\title{
Multiplying probe for accurate power measurements on an RF driven atmospheric pressure plasma jet applied to the COST reference microplasma jet
}

\author{
Citation for published version (APA): \\ Beijer, P. A. C., Sobota, A., van Veldhuizen, E. M., \& Kroesen, G. M. W. (2016). Multiplying probe for accurate \\ power measurements on an RF driven atmospheric pressure plasma jet applied to the COST reference \\ microplasma jet. Journal of Physics D: Applied Physics, 49(10), 1-12. [104001]. https://doi.org/10.1088/0022- \\ 3727/49/10/104001
}

Document license:

TAVERNE

DOI:

10.1088/0022-3727/49/10/104001

Document status and date:

Published: 08/02/2016

\section{Document Version:}

Publisher's PDF, also known as Version of Record (includes final page, issue and volume numbers)

\section{Please check the document version of this publication:}

- A submitted manuscript is the version of the article upon submission and before peer-review. There can be important differences between the submitted version and the official published version of record. People interested in the research are advised to contact the author for the final version of the publication, or visit the DOI to the publisher's website.

- The final author version and the galley proof are versions of the publication after peer review.

- The final published version features the final layout of the paper including the volume, issue and page numbers.

Link to publication

\footnotetext{
General rights

- You may freely distribute the URL identifying the publication in the public portal. follow below link for the End User Agreement:

www.tue.nl/taverne

\section{Take down policy}

If you believe that this document breaches copyright please contact us at:

openaccess@tue.nl

providing details and we will investigate your claim.
}

Copyright and moral rights for the publications made accessible in the public portal are retained by the authors and/or other copyright owners and it is a condition of accessing publications that users recognise and abide by the legal requirements associated with these rights.

- Users may download and print one copy of any publication from the public portal for the purpose of private study or research.

- You may not further distribute the material or use it for any profit-making activity or commercial gain

If the publication is distributed under the terms of Article $25 \mathrm{fa}$ of the Dutch Copyright Act, indicated by the "Taverne" license above, please 
Multiplying probe for accurate power measurements on an RF driven atmospheric pressure plasma jet applied to the COST reference microplasma jet

This content has been downloaded from IOPscience. Please scroll down to see the full text.

2016 J. Phys. D: Appl. Phys. 49104001

(http://iopscience.iop.org/0022-3727/49/10/104001)

View the table of contents for this issue, or go to the journal homepage for more

Download details:

IP Address: 131.155.151.8

This content was downloaded on 19/04/2016 at $11: 46$

Please note that terms and conditions apply. 


\title{
Multiplying probe for accurate power measurements on an RF driven atmospheric pressure plasma jet applied to the COST reference microplasma jet
}

\author{
P A C Beijer ${ }^{1}$, A Sobota ${ }^{2}$, E M van Veldhuizen ${ }^{2}$ and G M W Kroesen ${ }^{2}$ \\ ${ }^{1}$ Eindhoven University of Technology, Equipment and Prototype Center, Postbus 513, 5600MB \\ Eindhoven, The Netherlands \\ ${ }^{2}$ Eindhoven University of Technology, EPG, Postbus 513, 5600MB Eindhoven, The Netherlands \\ E-mail: a.sobota@tue.nl
}

Received 5 October 2015, revised 10 December 2015

Accepted for publication 30 December 2015

Published 8 February 2016

\begin{abstract}
In this paper a new multiplying probe for measuring the power dissipated in a miniature capacitively coupled, RF driven, atmospheric pressure plasma jet ( $\mu$ APPJ-COST Reference Microplasma Jet-COST RMJ) is presented. The approach aims for substantially higher accuracy than provided by traditionally applied methods using bi-directional power meters or commercially available voltage and current probes in conjunction with digitizing oscilloscopes. The probe is placed on a miniature PCB and designed to minimize losses, influence of unknown elements, crosstalk and variations in temperature. The probe is designed to measure powers of the order of magnitude of $0.1-10 \mathrm{~W}$. It is estimated that it measures power with less than $2 \%$ deviation from the real value in the tested power range. The design was applied to measure power dissipated in COST-RMJ running in helium with a small addition of oxygen.
\end{abstract}

Keywords: COST reference microplasma jet, RF, accurate power measurement, power probe

(Some figures may appear in colour only in the online journal)

\section{Introduction}

Atmospheric pressure plasma jets (APPJs) constitute a new generation of plasma sources that produce a non-thermal discharge at atmospheric pressure, often in air, featuring low current transfer and producing a cocktail of reactive species and radiation that can be used in biomedical applications as well as for surface modification [1-3]. They require high voltage and can be driven using sinusoidal or pulsed excitation in a range of frequencies from the $\mathrm{kHz}$ range to lowfrequency RF (several MHz). The topic of this paper is the COST Reference Microplasma Jet (COST-RMJ), the $\mu$ APPJ described in [4-16]. It is RF-driven, with the RF-powered and the grounded electrodes facing each other and gas flowing through the electrode gap $[4,5]$. The schematic is given in figure 1 .
The highly capacitive nature of this COST-RMJ makes the determination of RF power susceptible to errors in the voltage and current measurements. Reactive power circulating in the system is typically much larger in magnitude than the real dissipated power in the plasma. Hence, synchronicity deviations between voltage and current measurements in terms of phase and delay, as well as distortion and crosstalk will give rise to considerable errors. Reactive power tends to give false contributions to the result of real power calculation even for small imperfections in the voltage and current measurements. Moreover, the dynamic range of the current measurement is partly consumed by the current flowing in parasitic capacitance shunting the COST-RMJ. The latter is formed by stray and cable capacitance and, depending on its location, the capacitance of the voltage probe. This causes the reactive power in the system to become higher than based on the 


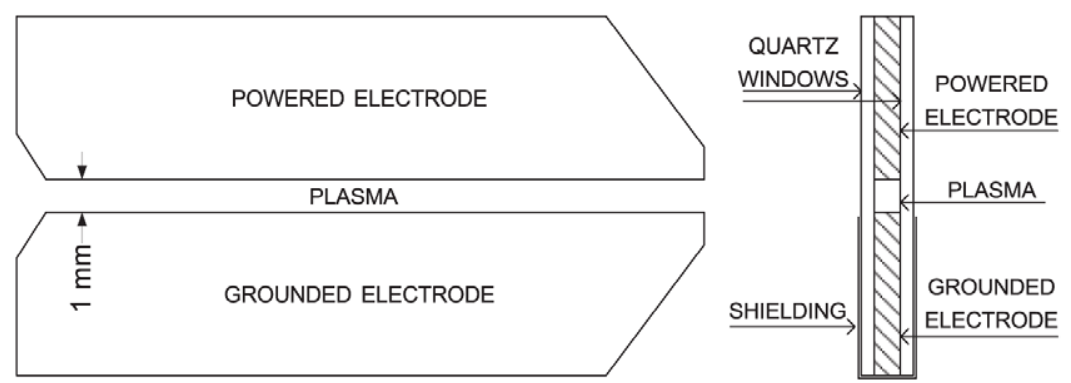

Figure 1. Schematics of the COST Reference Microplasma Jet-COST-RMJ [16] on the left hand side and its cross section on the right hand side. It consists of symmetric stainless steel electrodes $1 \mathrm{~mm}$ apart covered by quartz windows. The plasma will form in the volume $1 \mathrm{~mm}^{2}$ in cross section defined by the electrodes and the quartz windows.

electrical model of the plasma alone. Error margins of $\pm 42 \%$ [17] for the calculated power have been reported in previous studies.

This paper presents a new, dedicated miniaturized active voltage and current probe that can be integrated into the COST-RMJ itself in an attempt to improve the accuracy of measuring power. Due to its small dimensions and consequent limited impact on the geometry of the COST-RMJ, measurement invasiveness is mitigated. In addition, identical signal paths for both voltage and current measurement are provided for optimal synchronicity. Current and voltage signals obtained in this way can be used for power calculation by performing multiplication and averaging. To this end, a wide bandwidth analogue multiplier and averaging filter is used and integrated into the probe to avoid degradation of the voltage and current signals due to imperfect or unmatched cable connections. The resulting output provides a DC voltage showing the dissipated power in the plasma. In order to verify the accuracy and reproducibility of the proposed power measurement, as well as to ease the practical use of the probe a calibration method is enclosed where the measuring result and its accuracy can be traced back to a known resistive reference load and a voltage measurement.

\section{Identification of the problem}

In an RF-excited APPJ the determination of power is extremely sensitive to the phase angle between the voltage applied to the electrodes and the current. This phase angle departs from $90^{\circ}$ by only a few degrees depending on the setup, which makes it difficult to measure, yet an error of only $0.1^{\circ}$ results in $5 \%$ error in the calculated power. In this section the source of errors in conventional ways of measuring power in RF-driven APPJs will be discussed.

Figure 2 shows a circuit model of the COST-RMJ in a typical electrical test environment. An RF source complemented with an impedance matching network provides power for the COST-RMJ. Typically a frequency of $13.56 \mathrm{MHz}$ is used. The plasma is represented by the series connection of the electrode sheath capacitance $C_{s}$ and a resistor $R_{p}$ representing the bulk quasi-neutral region. The latter, being the only resistive element, is where power is being dissipated. The purpose of the matching network is to allow maximum power transfer from the RF source to the plasma. Ideally it makes the COST-RMJs complex impedance appear equal to the complex conjugate of the RF sources output impedance. $C_{\text {stray }}$ represents the inevitable parasitic capacitance determined by the geometry of the electrodes. The COST-RMJ is connected to the matching network with a cable of arbitrary length. Typically (but not necessarily) an asymmetrical coaxial cable is used where one electrode of the COST-RMJ is connected to the grounded outer conductor. Since the COST-RMJ cannot provide proper characteristic termination, this cable must be considered as an additional parasitic capacitance shunting the COST-RMJ. There is also the additional capacitance of the voltage probe.

Some variations on the diagram shown in figure 2 involve alternate locations of the voltage and current probes. In order to exclude the contribution of the current flowing in the voltage probe impedance from the current measurement, the sequence of the two probes may be reversed. This however may affect the voltage measurement due to the current probe impedance not being perfectly zero. The probes may also be placed after the cable directly at the terminals of the COST-RMJ. This avoids the inclusion of cable losses in the power measurement but it will often be difficult to eliminate the cable altogether because of bulkiness of the probes.

The voltage and current probes in the circuit provide signals from which the power dissipated in the plasma can be derived, where power is defined as the average product of voltage and current. This computation may be performed by e.g. a digital oscilloscope with math capabilities. While this method seems straightforward, the large ratio between reactive and real power however makes the result of real power calculation very susceptible to synchronicity errors between voltage and current measurements. These errors may be caused by differences in propagation time between $V$ and $I$ signals to the point of multiplication, but also differences in frequency and phase response or distortion properties may cause loss of orthogonality. This results in reactive power bleeding into the result of real power calculation, leading to considerable errors. Another cause may be crosstalk between the $V$ and $I$ signals leading to false (auto)correlation products after multiplication. Also finite immunity to common mode currents in the $V$ and $I$ measuring trajectories can give rise to false contributions.

The sensitivity to synchronicity errors is clearly illustrated by considering sinusoidal excitation of the COST-RMJ. In this special case, dissipated power is defined by: 


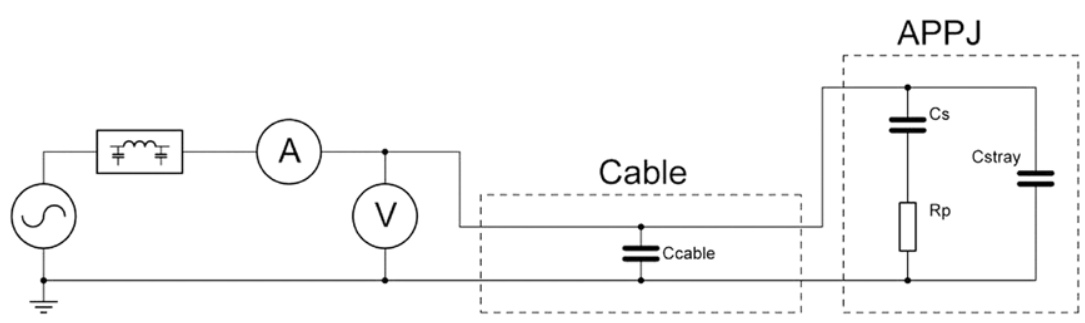

Figure 2. Circuit model of the COST-RMJ in a typical test environment.

$$
P=V_{\mathrm{rms}} I_{\mathrm{rms}} \cos \varphi,
$$

where $\varphi$ is the phase angle between voltage and current. Without plasma present and consequently no circuit element to dissipate any power, $\varphi=90^{\circ}$. Note that in this case it will be impossible to adjust the matching circuit for maximum power transfer. It is assumed that dielectric losses in the material from which the COST-RMJ body is constructed is negligible. Also radiated RF power is assumed to be negligible at the relevant frequencies of operation.

For the reference model COST-RMJ excited at $13.56 \mathrm{MHz}$ and purged with helium, the phase angle departs from $90^{\circ}$ by only a few degrees depending on the measuring setup and probe locations. The cosine function causes small absolute deviations in $\varphi$ to result in large relative deviations in calculated power. A measuring uncertainty of $0.1^{\circ}$ for example, results in $5 \%$ change in the computed power if $\varphi=88^{\circ}$. The phase error of $0.1^{\circ}$ will result from a mere $4 \mathrm{~mm}$ mismatch in propagation path lengths of $V$ and $I$ signals at $13.56 \mathrm{MHz}$.

Likewise, seemingly insignificant mismatches in frequency and phase response between $V$ and $I$ channels can introduce significant deviations in calculated power. This can be caused by differences between the analogue frontends of individual oscilloscope channels particularly when used with different attenuator settings. Theoretically these mismatches can be compensated for by calibrating the measurement system for a known impedance and frequency (assuming sinusoidal excitation). Practically however, small phase deviations may be caused by circumstances that are very difficult to control or replicate. This includes routing of cables, exact placement of the $V$ and $I$ probes, orientation of the COST-RMJ and proximity of conductive bodies. A common cause for crosstalk between $V$ and $I$ signals is coupling of the magnetic field associated with the circuit current, into the loop formed by the voltage probe and its ground lead. The induced EMF in this loop is added to the voltage measurement leading to false autocorrelation products after multiplication with the current signal. Similarly voltage information may be picked up by the current probe due to unwanted capacitive coupling. The coupling factors for these crosstalk components are highly dependent on parasitic properties determined by placement, proximity effects, surface area and orientation of loops, etc.

\section{Multiplying probe}

A multiplying probe has been designed to increase accuracy of power measurement in the COST-RMJ and potentially similar
RF-driven devices. The general principle of operation depends on determining the average product of voltage and current in the plasma as depicted in figure 3. In the following subsections individual parts of the probe will be described, while the whole system can be seen in the schematics presented in the figure 13. Throughout the paper the term 'grounded electrode' will be used for the electrode connected to ground through a sensing resistor.

\subsection{Voltage and current measurement}

This section presents measures that were put in place to attain identical transfer characteristics for $V$ and $I$ measurements over a frequency range as wide as possible.

The voltage across the electrodes is attenuated by the capacitive divider $C_{a}$ and $C_{b}$ as presented in figure 3 (top) in order to bring it to a level that can be handled by subsequent electronic processing. It is then applied to a high input impedance buffer amplifier, whose purpose is to minimize the load on the capacitive divider. This provides a flat frequency response extending to frequencies several orders of magnitude below the frequencies of interest while keeping the values of the capacitors in the divider low. The latter is important to minimize the amount of additional capacitance shunting the plasma jet.

More detail is given in the electronic circuit diagram in figure 4, where it is shown that the capacitive divider is implemented in two stages by capacitors $\mathrm{C} 1-\mathrm{C} 3$ and $\mathrm{C} 6-\mathrm{C} 7$. The additional capacitance shunting the plasma jet is largely determined by the value of $\mathrm{C} 1$ and in this case set to $0.5 \mathrm{pF}$. U2a is a Texas Instruments OPA653 fixed gain JFET amplifier configured for a gain of 2. R4 provides DC bias to its input and also defines the load as seen by the capacitive divider thereby determining the lower cut-off frequency.

Current is measured by means of sense resistor $R_{\text {sense }}$ in the grounded electrode connection in figure 3 , and the signal is buffered by a high input impedance amplifier identical to the one used in the voltage measurement. $R_{\text {sense }}$ is replaced by $R_{1}$ in the circuit diagram on figure $4 . R_{1}$ is shunted by the parasitic capacitance formed by the shield that was introduced around the grounded electrode, as indicated in figure 3 and shown in the photograph of the entire system on figure 5 . The purpose of this shield is to make the parasitic capacitance $C_{\text {par }}$ in figure 3 (bottom) a fixed value. Without the shield $C_{\text {par }}$ depends strongly on external factors which are difficult to control like proximity effects and orientation of the jet. Ideally only the surface directly facing the powered electrode should be left exposed. 

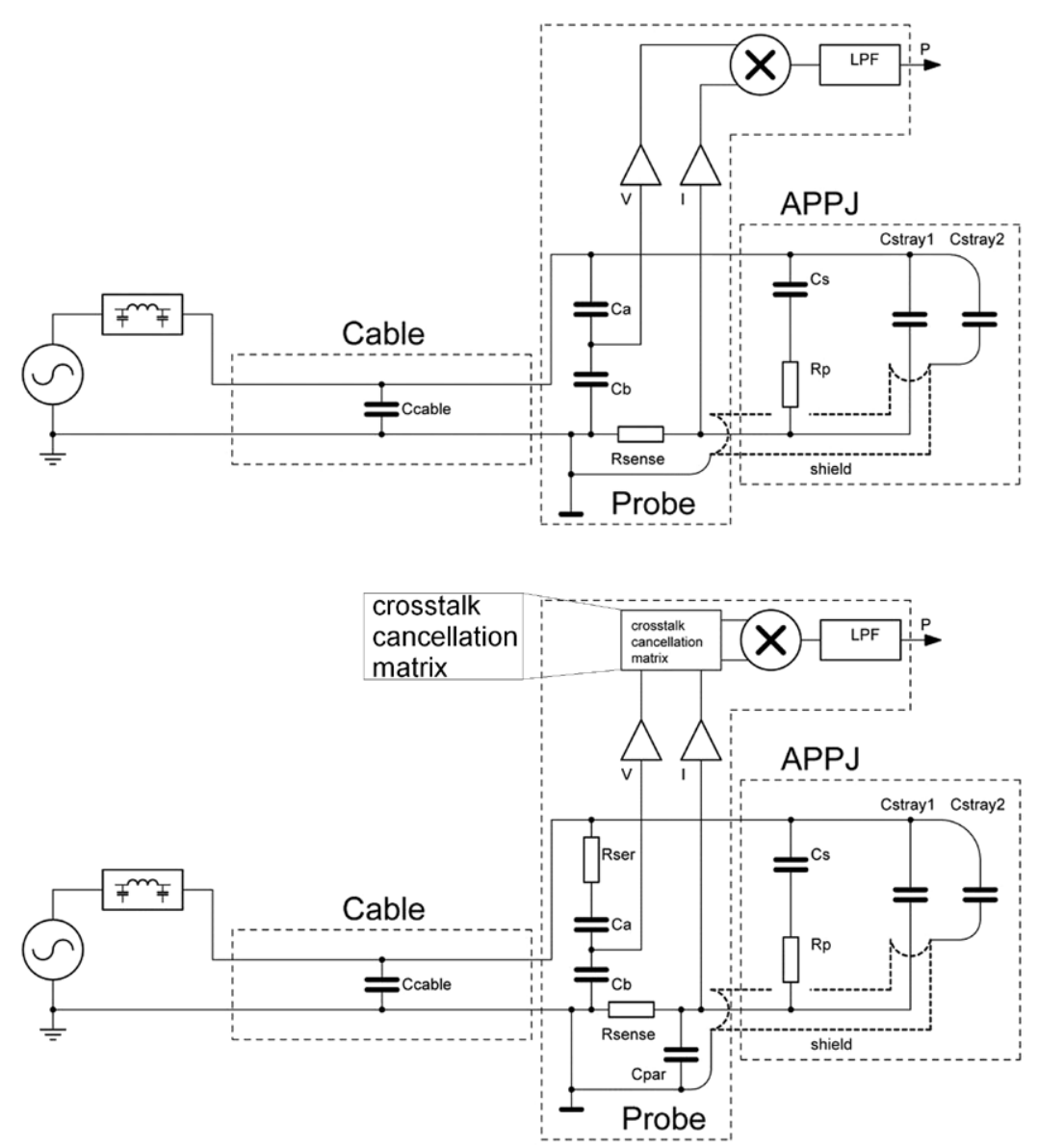

Figure 3. Block diagram of the multiplying probe system (top) with error compensation (bottom). Details of individual parts of the probe are described in the text and shown in more detail in subsequent figures showing electronic circuit diagrams.

Keeping $C_{\text {par }}$ constant is important since the time constant $\tau=C_{\text {par }} R_{\text {sense }}$ introduces a pole (singularity) in the transfer characteristic of the current measurement, which causes a small phase shift in the measured current. Having the current pole at a fixed position makes it possible to match the transfer characteristic of the voltage measurement path to it. This is accomplished by adding resistor $R_{\text {ser }}$ to the capacitive voltage divider network as shown in figure 3 (bottom). The time constant formed by $R_{\text {ser }}$ and the series connection of $C_{a}$ and $C_{b}$ must be equal to $C_{\mathrm{par}} R_{\text {sense. }}$ In the electronic circuit diagram in figure $4, \mathrm{R} 2$ is given instead of $R_{\text {ser }}$ to make this adjustment. Further fine adjustment of time constant $\tau=C_{\mathrm{par}} R_{\text {sense }}$ is provided by means of trimmer capacitor $C_{33}$ in figure 4 and later indicated on the probe in figure 7. The adjustment of this capacitor is done during the calibration and adjustment procedure explained later in the text in section 4.

Another role of the resistor R2 is to compensate for small phase differences, due to temperature differences between the two amplifiers U1a and U2a in figure 4 caused by differing signal levels. Finding a suitable dual amplifier in one chip might be a potential future improvement for the probe.

The value of coupling capacitor $C_{4}$ in figure 4 is chosen such that the lower cut-off frequency caused by bias resistor $R_{3}$ and $C_{4}$ closely matches the cut-off frequency of the voltage measurement path.
Since the shield partly covers the grounded electrode of the jet, it also reduces capacitance $C_{\text {stray }}$ between the grounded and powered electrode. Effectively $C_{\text {stray }}$ is split in two parts: $C_{\text {stray } 1}$ and $C_{\text {stray2 }}$ as schematically shown in figure 3 . Reactive current flowing in $C_{\text {stray } 2}$ is diverted directly to ground without passing through the current sense resistor $R_{\text {sense. This significantly }}$ reduces the amount of reactive current seen by the current measurement and hence allowing for better accuracy of the measurement. In terms of phase shift between $V$ and $I$ signals it means that it departs further from $90^{\circ}$ for the same power level.

Note that reducing the inclusion of reactive current in the measurement by splitting $C_{\text {stray }}$ by means of shielding of the grounded electrode like shown in figure 5 will only have effect for those areas of the electrode not exposed to the plasma. In a coaxial plasma jet where the powered electrode is completely enclosed by the grounded electrode the shielding will not change $C_{\text {stray. }}$.

The output signals of buffer amplifiers U1a and U2a in figure 4 represent the current and voltage parameters respectively, measured at the point where the probe is inserted. In order to optimally utilize the ADL5391 analog multiplier to produce the power signal, which will be addressed later and is a part of the schematics shown in figure 8, the single-ended voltage and current signals are subsequently converted to symmetrical differential signals by Analog Devices ADA4950-2 


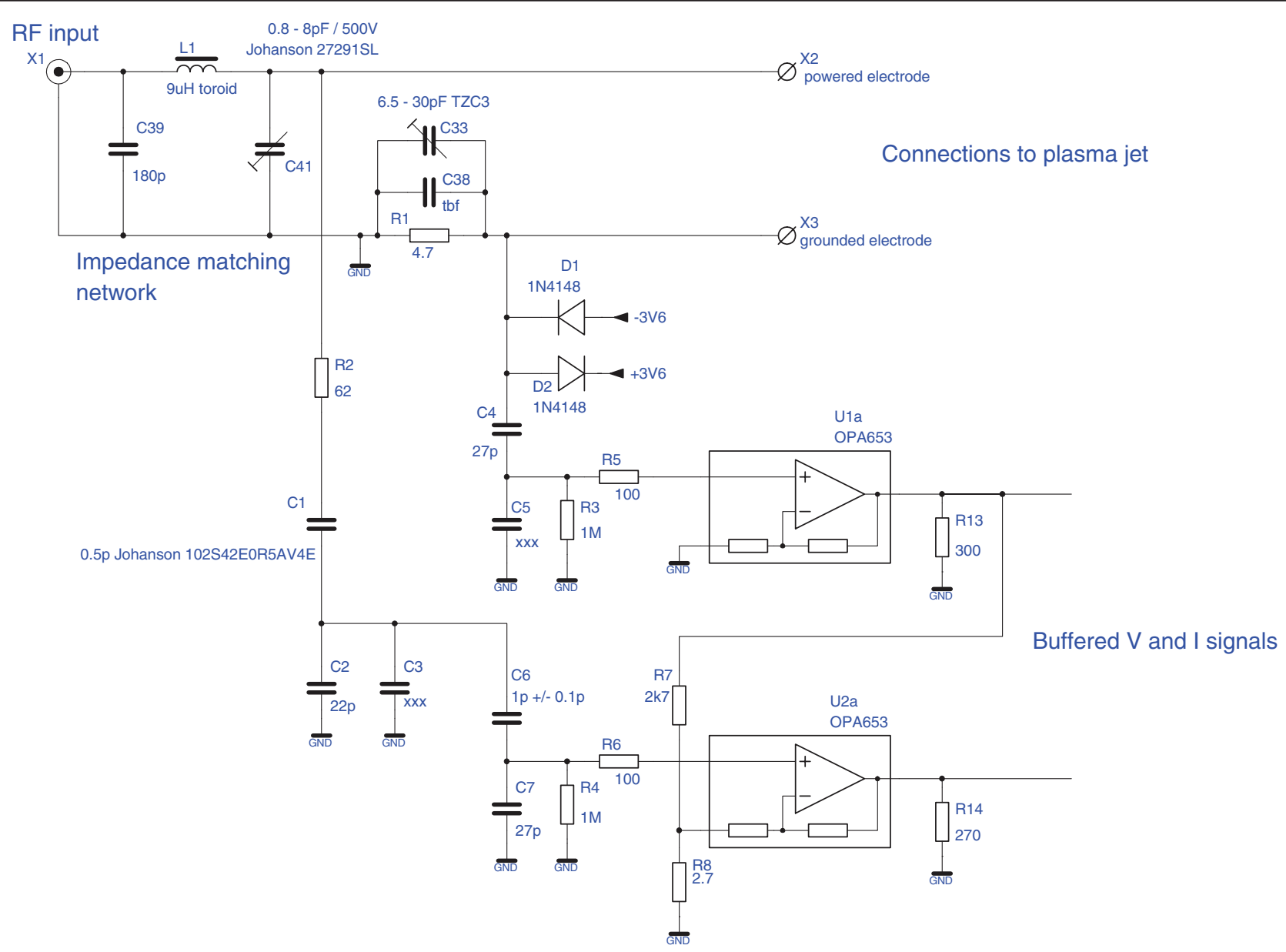

Figure 4. Electronic circuit diagram of the capacitive divider.

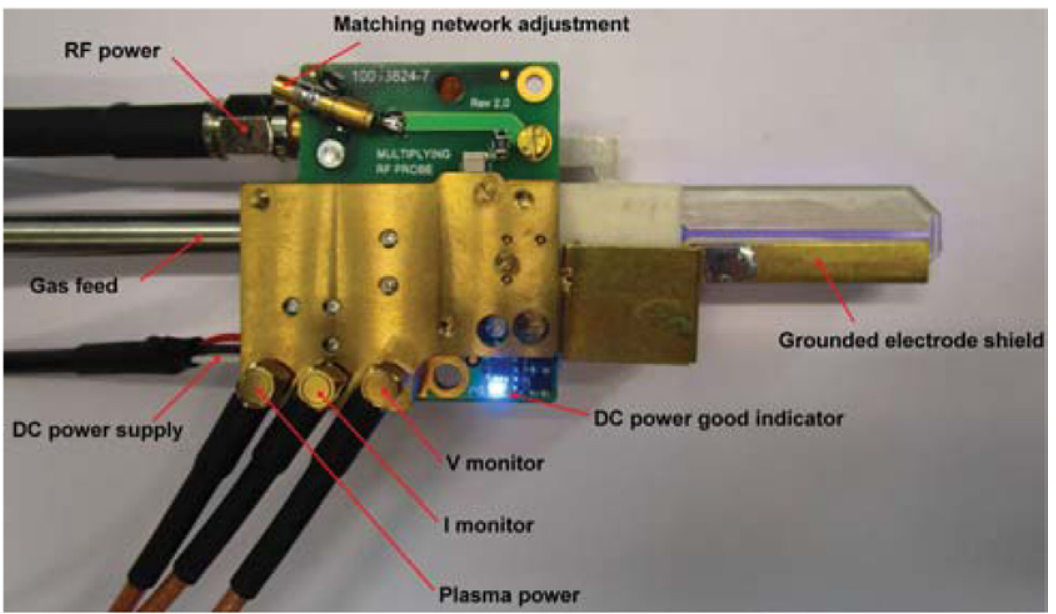

Figure 5. Photograph of the plasma jet with shielded grounded electrode and incorporated power measurement system.

dual differential amplifiers (U3) shown in figure 6 as a part of the crosstalk cancellation matrix, described in the following subsection.

\subsection{Differential amplifiers and crosstalk cancellation}

In the process of generating the $V$ and $I$ signals until the point where multiplication takes place it is unavoidable that some voltage information gets picked up by the current signal path and vice versa. These crosstalk phenomena may be minimized by careful PCB layout design, but since they are caused by inherent parasitics they can never be fully eliminated.

The crosstalk phenomena may be initiated by parasitic capacitive coupling between circuit nodes and magnetic coupling between current conducting loops. In addition, signal return currents flowing through shared ground conductors can give rise to crosstalk due to parasitic self-inductance or resistance of that conductor. 


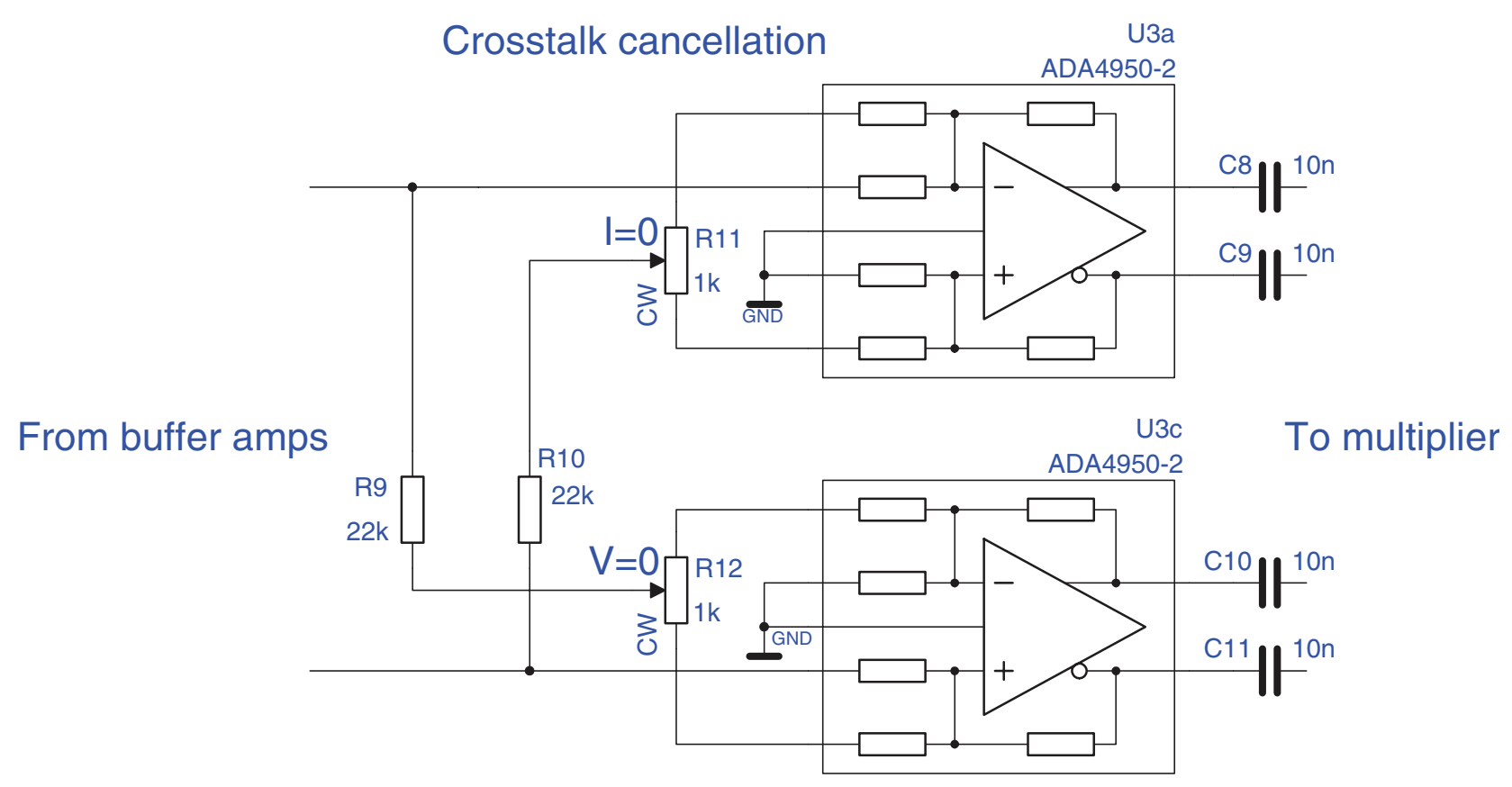

Figure 6. Electronic circuit diagram of the differential amplifier and crosstalk cancellation matrix.

For a single frequency the transfer function from the actual plasma voltage and current to the point where multiplication takes place can be formulated in matrix form:

$$
\left(\begin{array}{l}
S_{v} \\
S_{i}
\end{array}\right)=\left(\begin{array}{ll}
a & b \\
c & d
\end{array}\right)\left(\begin{array}{l}
v \\
i
\end{array}\right)=\left(\begin{array}{l}
a v+b i \\
c v+d i
\end{array}\right)
$$

where $v$ and $i$ are the plasma voltage and current in complex representation and $S_{v}$ and $S_{i}$ are the signals representing measured voltage and current at the point of multiplication including crosstalk components. Note that since the signals at the input of the multiplier are both in the form of voltages, $b$ and $d$ have the dimension of impedance whereas $a$ and $c$ are dimensionless. Parameters $a, b, c$ and $d$ represent complex matrix coefficients which may also be frequency dependent.

Average power in the plasma is given by $|v \| i| \cos \varphi$ which is equal to the dot product of vectors $v$ and $i$ :

$$
P=v \cdot i=v_{\operatorname{Re}} i_{\operatorname{Re}}+v_{\operatorname{Im}} i_{\mathrm{Im}}
$$

Another way to define power is as the running average of the arithmetic product of voltage and current in the time domain. This is the method applied in the proposed probe design. This implies that the averaged result of time domain multiplication of two vectors is proportional to the dot product of these vectors. Due to the aforementioned effects of parasitics and crosstalk phenomena the actual inputs to the multiplying operation are $S_{v}$ and $S_{i}$ rather then $v$ and $i$ and can be written as:

$$
\begin{aligned}
S_{v}= & \left(a_{\mathrm{Re}} v_{\mathrm{Re}}-a_{\mathrm{Im}} v_{\mathrm{Im}}+b_{\mathrm{Re}} i_{\mathrm{Re}}-b_{\mathrm{Im}} i_{\mathrm{Im}}\right) \\
& +j\left(a_{\mathrm{Im}} v_{\mathrm{Re}}+a_{\mathrm{Re}} v_{\mathrm{Im}}+b_{\mathrm{Im}} i_{\mathrm{Re}}+b_{\mathrm{Re}} i_{\mathrm{Im}}\right) \\
S_{i}= & \left(c_{\mathrm{Re}} v_{\mathrm{Re}}-c_{\mathrm{Im}} v_{\mathrm{Im}}+d_{\mathrm{Re}} i_{\mathrm{Re}}-d_{\mathrm{Im}} i_{\mathrm{Im}}\right) \\
& +j\left(c_{\mathrm{Im}} v_{\mathrm{Re}}+c_{\mathrm{Re}} v_{\mathrm{Im}}+d_{\mathrm{Im}} i_{\mathrm{Re}}+d_{\mathrm{Re}} i_{\mathrm{Im}}\right)
\end{aligned}
$$

Meticulous matching of the $V$ and $I$ signal paths translates in vectors $a$ and $d$ having the same argument for any given frequency. This is accomplished by careful PCB layout design, using identical components in the signal paths, having equal propagation lengths and applying compensation for parasitic time constants as described earlier.

$$
\arg (a)=\arg (d) \Longrightarrow a_{\mathrm{Re}} d_{\mathrm{Im}}=a_{\mathrm{Im}} d_{\mathrm{Re}}
$$

The averaged result of multiplication now has the following form:

$$
\begin{aligned}
& S_{v} \cdot S_{i}=S_{v, \operatorname{Re}} S_{i, \operatorname{Re}}+S_{v, \operatorname{Im}} S_{i, \operatorname{Im}} \\
& =\left(a_{\mathrm{Re}} d_{\mathrm{Re}}+b_{\mathrm{Re}} c_{\mathrm{Re}}+a_{\mathrm{Im}} d_{\mathrm{Im}}+b_{\mathrm{Im}} c_{\mathrm{Im}}\right)(v \cdot i) \\
& +\left(a_{\mathrm{Re}} c_{\mathrm{Re}}+a_{\mathrm{Im}} c_{\mathrm{Im}}\right)\left(v_{\mathrm{Re}}^{2}+v_{\mathrm{Im}}^{2}\right) \\
& +\left(b_{\mathrm{Re}} d_{\mathrm{Re}}+b_{\mathrm{Im}} d_{\mathrm{Im}}\right)\left(i_{\mathrm{Re}}^{2}+i_{\mathrm{Im}}^{2}\right) \\
& +\left(b_{\mathrm{Im}} c_{\mathrm{Re}}-b_{\mathrm{Re}} c_{\mathrm{Im}}\right)\left(i_{\mathrm{Re}} v_{\mathrm{Im}}-i_{\mathrm{Im}} v_{\mathrm{Re}}\right) \text {. }
\end{aligned}
$$

The first term represents a scalar value proportional to the desired dot product of $v$ and $i$ and thus proportional to average power. The second and third term are unwanted crosstalkinduced contributions proportional to the square of voltage and current respectively.

To realize the cancellation of all unwanted terms in equation (6) thereby eliminating contributions of reactive power, one has to set the real parts of $|b|$ and $|c|$ as follows:

$$
\begin{aligned}
& c_{\mathrm{Re}}=-\frac{a_{\mathrm{Im}} c_{\mathrm{Im}}}{a_{\mathrm{Re}}} \\
& b_{\mathrm{Re}}=-\frac{b_{\mathrm{Im}} d_{\mathrm{Im}}}{d_{\mathrm{Re}}} .
\end{aligned}
$$

The remaining result of the averaged multiplication is now solely proportional to $v \cdot i$.

In practice, the adjustment of $b_{\mathrm{Re}}$ and $c_{\mathrm{Re}}$ is provided by a deliberate adjustable amount of cross-coupling between voltage and current signal paths by means of trimmer potentiometers $R_{11}$ (voltage to current) and $R_{12}$ (current to voltage) shown in the schematics in figure 6. Each of them is placed on one input of the previously mentioned ADA4950 differential 

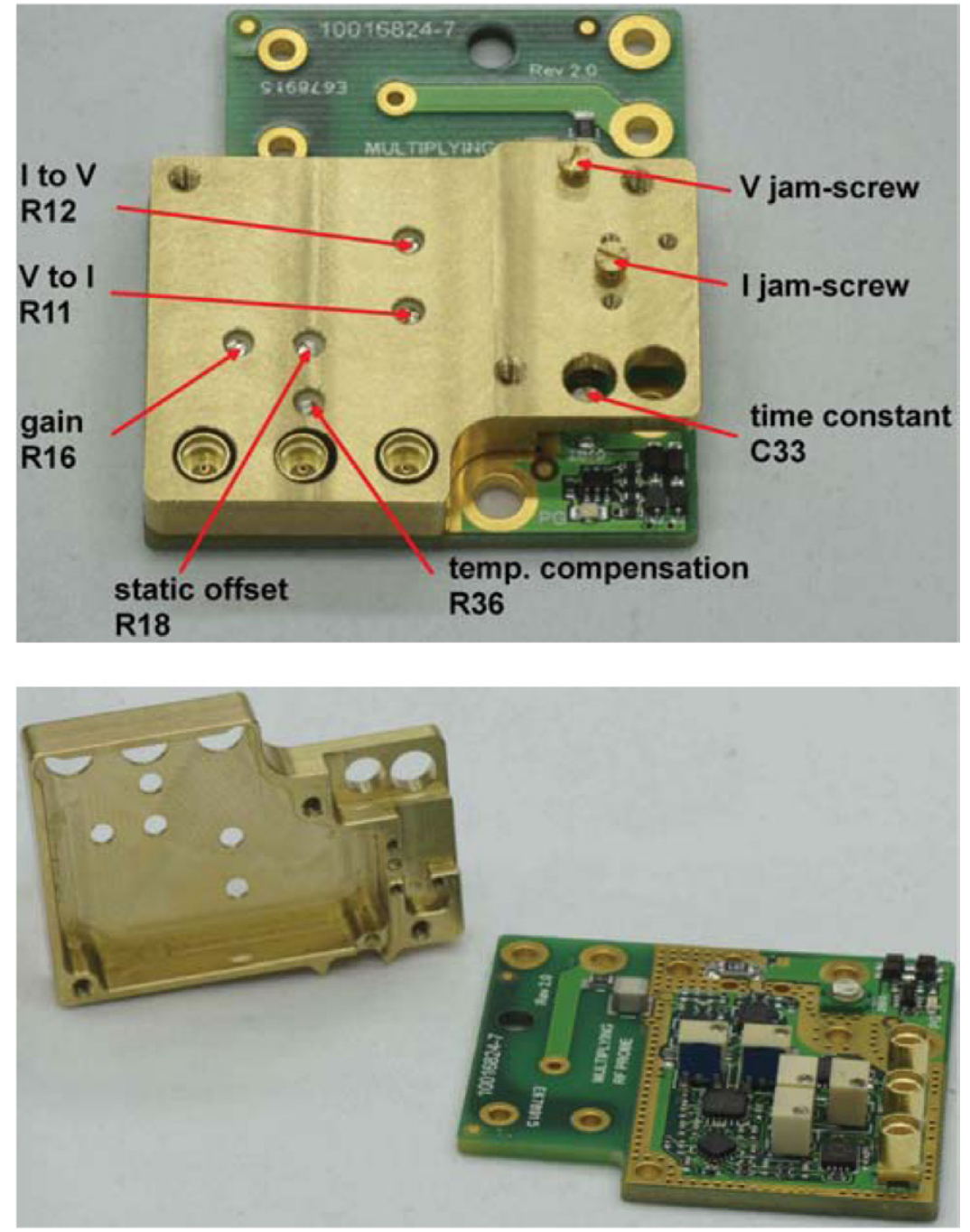

Figure 7. Photograph of the power measurement probe with indicated adjustment screws (top) and the probe with the shielding removed (bottom).

amplifiers in figure 6 that feature dual differential inputs for each channel, enabling the summation of signals. While one input is used for the main signal path (voltage or current), the second input can be used for adding a small adjustable amount of cross-coupling provided by $R_{11}$ and $R_{12}$. Since the adjustments can only be used to change the magnitude and sign of the cross-coupling but not its phase angle, they can be regarded as the manipulation of the real parts of parameters $b$ and $c$.

In the proposed probe design the measured $V$ and $I$ signals can be shorted individually at the exact point where they enter the probe by means of small jam-screws as indicated in figure 7. By doing so, the contribution of either voltage or current can be eliminated from the multiplication result, leaving only the square term of the other channel. This enables the adjustment of $b_{\mathrm{Re}}$ and $c_{\mathrm{Re}}$ by means of trimmer potentiometers R12 and R11 respectively in a way described later in section 4.

Since the $V$ and $I$ signal paths are designed to have a large bandwidth, parameters $a$ and $d$ are predominantly real for the frequencies of interest. Additionally, when practicing good general design techniques, the crosstalk between channels can typically be kept at least several orders of magnitude lower than the signal itself. This means that the magnitudes $|b|$ and $|c|$ are much smaller than the magnitudes of $|d|$ and $|a|$ respectively. Consequently, only a very small amount of deliberate cross-coupling needs to be applied in order to eliminate the square terms.

Note that this derivation is based on single frequency excitation of the plasma in which case the voltage and current can be represented as complex vectors. Since the matrix coefficients are generally frequency dependent, perfect cancellation can only be achieved at one single frequency. It is therefore important to perform the crosstalk adjustment at the plasma jets frequency of operation (typically $13.56 \mathrm{MHz}$ ). Non-linear behavior in the plasma may result in harmonic distortion of voltage and current, revealed by non-sinusoidal waveforms. Though the running average voltage and current arithmetic product definition for power is still valid in this case, small errors may arise due to imperfect crosstalk cancellation for the harmonics.

Another essential note must be made: the crosstalk cancellation mechanism works by virtue of the matrix coefficients being predictable and reproducible. In the probe design this is 


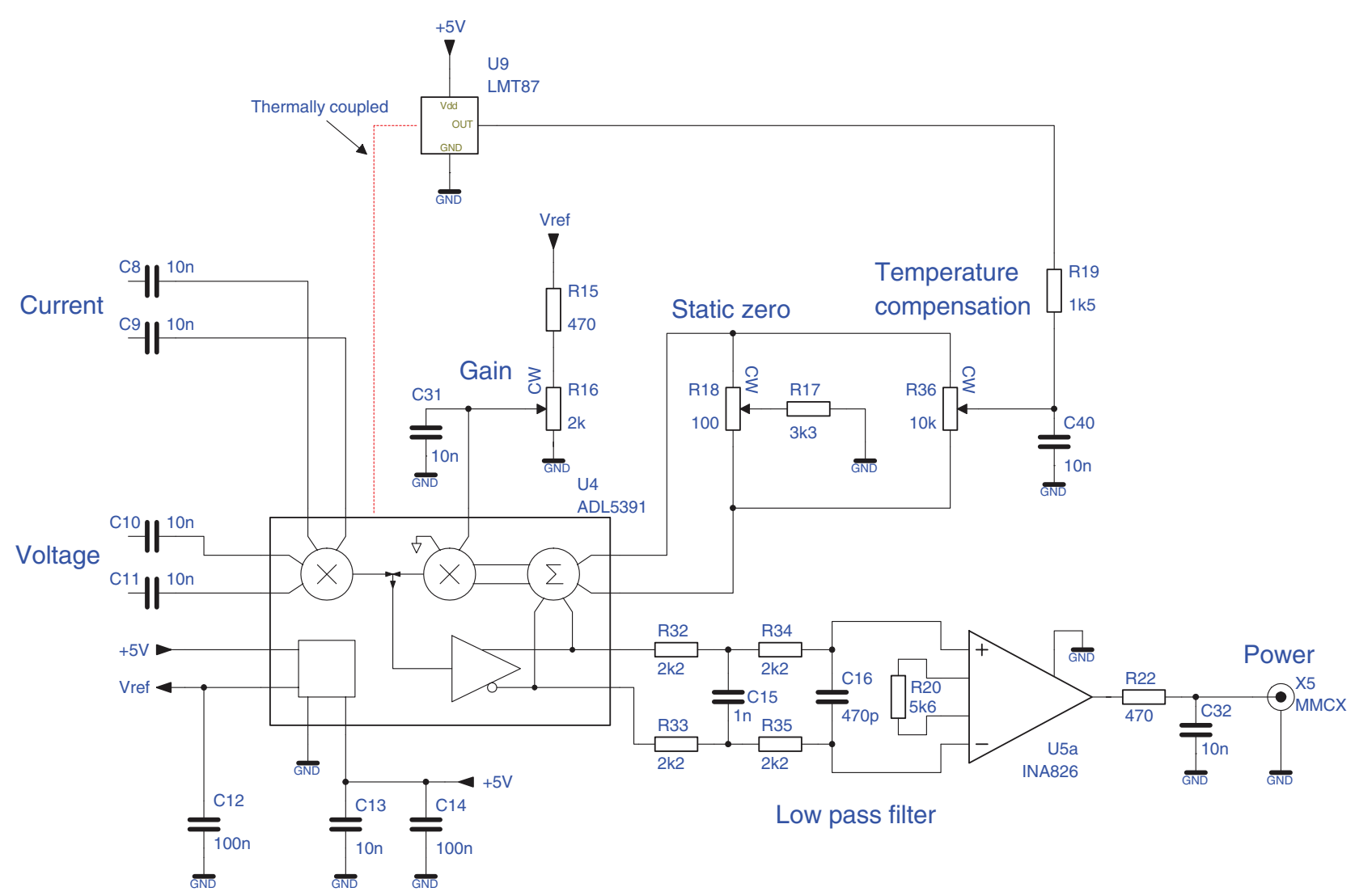

Figure 8. Electronic circuit diagram of the analog multiplier with temperature compensation system.

achieved by integrating the entire signal processing including multiplication onto a carefully designed miniaturized PCB. This and the fact that the probe is made an integral part of the plasma jets mechanical construction eliminates all dimensional variations and therewith variations in electrical parasitics. The circuit is also completely enclosed by shielding, as is visible in figures 5 and 7 (not to be confused by the shielded grounded electrode) in order to avoid unwanted and variable couplings from the surrounding environment.

\subsection{Analog multiplier}

The differential signals representing $S_{v}$ and $S_{i}$ are presented to the inputs of an Analog Devices ADL5391 analogue multiplier (U4) as shown in figure 8. This device features wide bandwidth $(2 \mathrm{GHz})$ and excellent symmetry between the two differential inputs. The output of the multiplier represents the instantaneous arithmetic product of its two input signals, the DC content of which is proportional to the average power to be measured. In order to extract the DC content and convert the differential output signal of the multiplier to a single ended signal, a second order low-pass filter (R32-R35, C15, C16) and an instrumentation amplifier (U5) have been implemented. The output available at connector X5 can be applied to a voltmeter in order to display the power level. The output level has been calibrated to $100 \mathrm{mV} \mathrm{W}^{-1}$. (Adjustable by means of trimmer potentiometer R16-details in section 4).

The static offset of the multiplier (i.e. its DC output with both inputs zero) can be adjusted to zero by means of trimmer R18 that is also indicated in figure 7.
The DC output of the multiplier exhibits a temperature dependency. In order to compensate for this, a temperature sensor is provided (U9) that is thermally coupled to the multiplier chip. The output signal of the sensor is applied to the offset control input of the multiplier via trimmer R36, also indicated in figure 7. Since both static offset adjustment and temperature compensation use the same input of the ADL5391, the adjustments have some mutual dependency and the adjustment may therefore require a few iterations.

\subsection{Waveform monitor outputs}

In order to view the current and voltage waveforms on an oscilloscope, two monitor outputs have been provided, as shown in figure 9. The monitor outputs are designed for $50 \mathrm{Ohm}$ termination.

The electronic circuit diagram of the entire probe is shown at the end of the article in figure 13.

\section{Calibration and adjustment}

Calibration and adjustment is necessary for a proper power measurement, and will be described in detail in the following list of steps:

(i) Calibration and adjustment of the probe starts with connecting the dedicated DC power supply and allowing the probe to warm up and thermally settle for $15 \mathrm{~min}$. The blue LED (the 'power good indicator' in figure 5) indicates that both positive and negative supply voltages are present. 


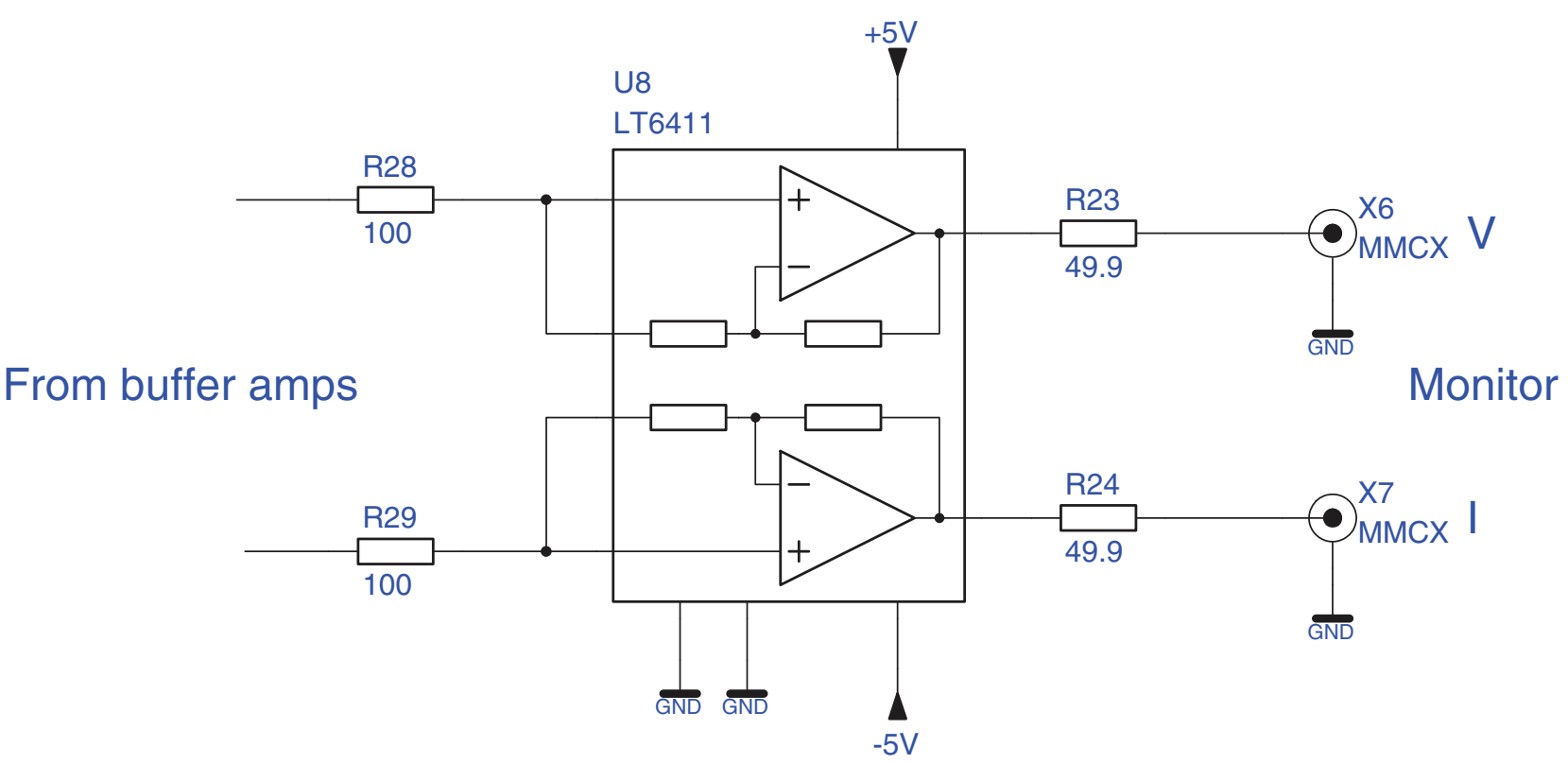

Figure 9. Electronic circuit diagram of the voltage and current waveform monitor outputs of the power probe. LT6411 is an off-theshelf electrical component. All the entrances to this component, including $+/-5 \mathrm{~V}$ and two times GND, are physical pins present on this component.

(ii) The first adjustment to be made is the static offset (R18) shown in figure 7 . For the adjustment of all trimmers a non-conductive plastic trimmer key must be used. Without RF power applied, R18 must initially be adjusted for $0 \mathrm{mV}$ at the power output ( $\mathrm{X} 5$ in figure 8 and 'Power' in figure 5).

(iii) The next step is adjusting the temperature compensation with R36 (figure 7) in order to minimize drift of the output voltage as a function of temperature. Small temperature variations can be imposed by gently modulating the airflow around the probe (for example by means of switching on and off a small fan). R36 can then be adjusted until these temperature variations have minimal effect on the output voltage. At a certain position of R36 it can be noticed that the correlation between imposed temperature variation and resulting change in output voltage changes sign. This is exactly the point where the overall temperature dependency is minimal. This adjustment procedure has a fairly heuristic character and may need to be improved in the future. The temperature compensation adjustment will also affect the static offset. Therefore some iterations of both adjustments may be required.

(iv) The next step is the adjustment of the crosstalk correlation. To this end RF signal must be applied to the plasma jet. No purging gas must be supplied at this stage in order to avoid plasma generation, but the voltage level across the electrodes should be sufficient to generate plasma if gas would be supplied. Next, the jam-screw to short the current signal (the 'I jam screw' in figure 7) must be tightened (finger tight). Now the output voltage of the probe can be adjusted to zero using trimmer R11, where R11 determines the amount of cross-coupling from $V$ to $I$. Following this operation, the current jam-screw can be released. (v) The same procedure must be repeated for the voltage jam-screw and R12 in order to adjust the cross-coupling from $I$ to $V$.

(vi) Next is the adjustment of the current sense resistor time constant. With both jam-screws released the output voltage must be set to zero using trimmer capacitor C33 (figure 7).

(vii) Subsequently the sensitivity of the voltage monitor output must be determined. To this end a calibrated voltage probe is externally connected to the jets RF input signal. This probe is connected to one channel of an oscilloscope with measurement facilities. A second channel of the oscilloscope is connected to the voltage monitor output properly terminated with $50 \mathrm{ohms}$. Now RF signal is applied to the jet. The measurement facilities of the oscilloscope can now be used to determine the ratio between electrode voltage and its representation at the monitor output. Alternatively an RF voltmeter may be used instead of the oscilloscope.

(viii) Finally the sensitivity of the probe can be calibrated. To this end an unpopulated reference load PCB (reference load PCB without the chip resistors, shown as 'empty PCB' in figure 10) must first be connected to the jet. With $\mathrm{RF}$ signal present C33 in figure 7 must be re-adjusted for zero output. This re-adjustment is needed because connecting the PCB slightly changes $C_{\mathrm{par}}$ shown in figure 3 and therewith the time constant. Next the populated reference PCB must be connected (reference load PCB with the chip resistors, shown as ' $100 \mathrm{k}$ load' in figure 10). With RF signal applied, the power in the reference load can now be calculated from the measured RMS voltage at the monitor output and its relation to electrode voltage and the known Ohmic value of the load (100 kOhm). Subsequently, trimmer R16 (gain adjustment in figure 7) 


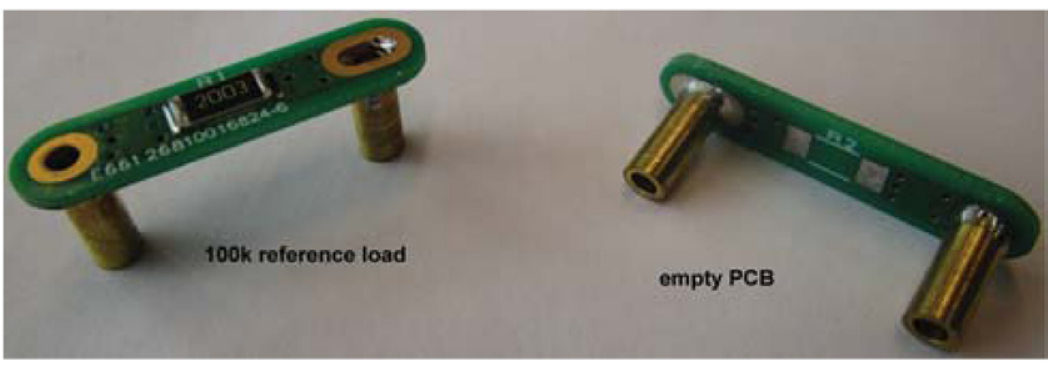

Figure 10. Photograph of the reference loads used for calibration and adjustment.

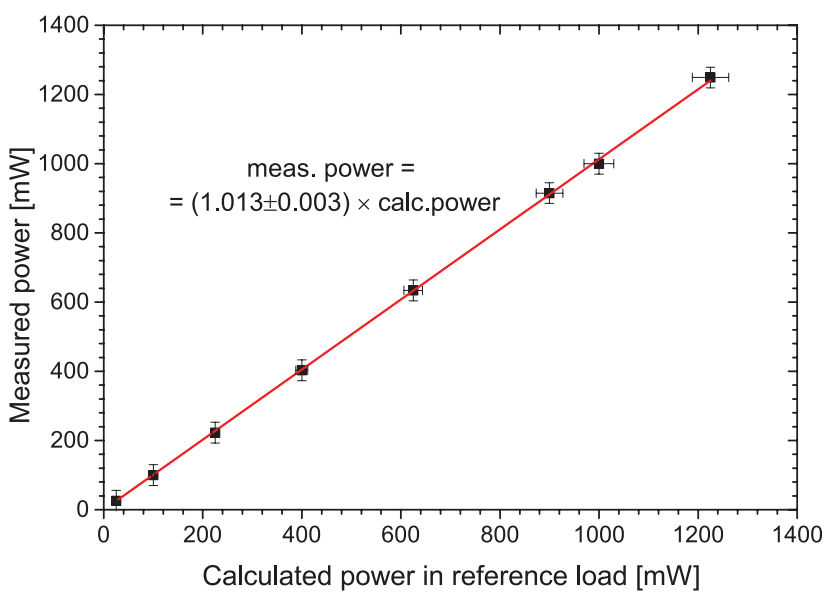

Figure 11. Measured power as a function of calculated input power. The input power was calculated from the monitored voltage and known reference load. The fit shown in red has been obtained by using the least squares method using a linear model without the constant term. The slope has been determined at the value of $1.013 \pm 0.003$, which means that the measured power is $1.3 \%$ larger than the applied (calculated) power, with the accuracy of $0.3 \%$.

can be set in order to have a DC value at the power output of the probe representing $100 \mathrm{mV} \mathrm{W}^{-1}$. After disconnecting the reference load, C33 must again be re-adjusted (repeat step 6).

Figure 11 shows the result of the calibration and adjustment procedure. It shows the measured power at different, well-known input powers. The results show that the measured power is linear with respect to the input power, and after the calibration and adjustment procedure mirrors the value of the input power with less than $2 \%$ error.

The device used to measure the vertical scale of figure 11 was a Keithley 2000 multimeter. It measured the DC output voltage of the power probe representing plasma power. According to the specifications of the meter the accuracy is $50 \mathrm{ppm}$ of reading $+35 \mathrm{ppm}$ of range in the used measuring range. Hence, the worst case relative error occurs at the lowest measured point which is then $2.6 \mathrm{mV} \pm 0.0036 \mathrm{mV}$. The error is much smaller than the actual digits that were recorded and hence the error introduced by the multimeter is negligible. The accuracy of how well the output voltage mirrors the plasma-power is mainly determined by the power probe itself. During the measurements with no RF power applied the probe's output voltage fluctuated by $3 \mathrm{mV}$ around zero. Using that value we estimated the accuracy at $30 \mathrm{~mW}$, which represents the vertical error bar in figure 11 .

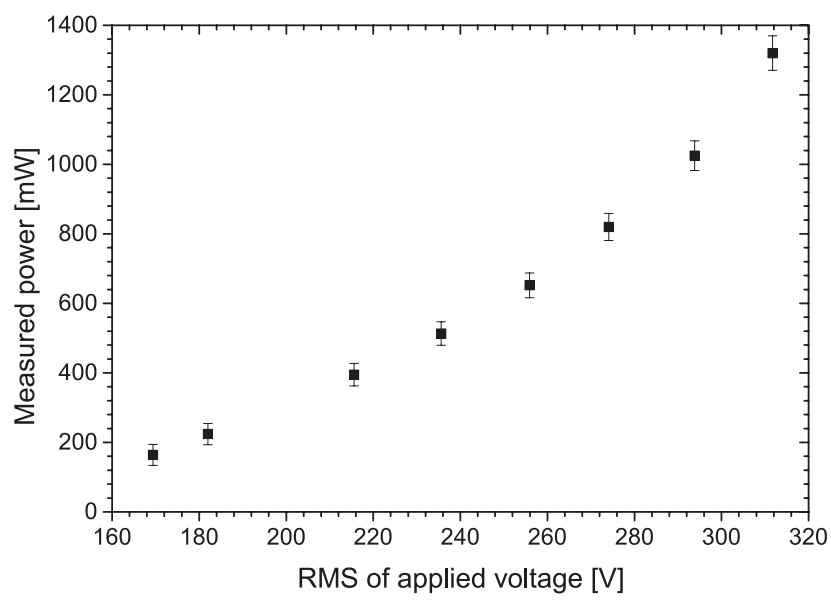

Figure 12. Power dissipated in a COST-RMJ driven in $5.0 \mathrm{He}$ with addition of $0.5 \%$ of $\mathrm{O}_{2}$.

Voltage for the calculated power in the reference load was measured with an oscilloscope (TDS524A) capable of calculating RMS values and a voltage probe Tektronix P6139A with an attenuation factor of 10 . The specifications state a vertical accuracy of $1 \%$, without information on how the vertical accuracy error propagates into the RMS result. To characterize this value a reference measurement was performed where a $1 \mathrm{kHz}$ sine signal was connected to the oscilloscope and had it calculate the RMS value. Then we connected the same $1 \mathrm{kHz}$ signal to the Keithley and measured the RMS value. The results were the same within $0.3 \%$. We are therefore confident that the $1 \%$ accuracy is a safe estimate for the RMS voltage value. Consequently, for the calculated power on the horizontal axis the error bars are set at 3\%, taking into account $1 \%$ tolerance of the reference load.

This part of the measurement could be made arbitrarily more accurate by using better equipment but it is not fundamental to the power probe.

At this time gas can be supplied and the probe is ready to measure the power dissipated in the generated plasma.

\section{Power measurement in a COST-RMJ in helium}

Using a range of input voltages and a flow of He of 5.0 purity with an admixture of $0.5 \%$ oxygen, accurate power measurements have been performed, shown in figure 12. A recent study on power coupling and electrical characterization of this source [15] provided a set of power measurements that compare quite well with the set provided in this paper. In [15] 


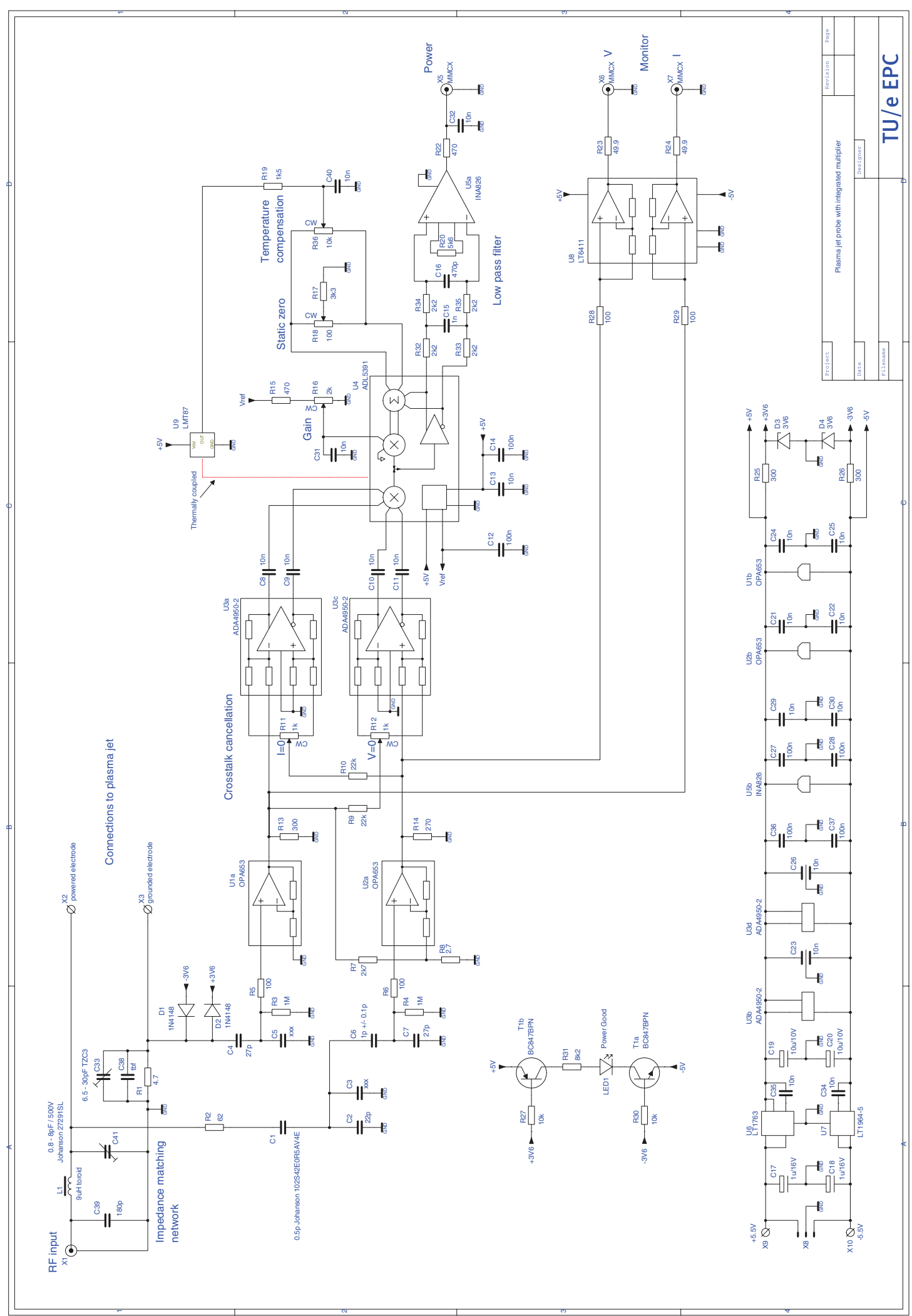

Figure 13. Electronic circuit diagram of the entire probe. The 'power good' LED indicates that the probe and the jet are operational. The mark $3 \mathrm{~V} 6$ signifies $3.6 \mathrm{~V}$.

the power coupling between the RF power generator and the jet was based on a series $L C$ resonance with the discharge gap acting as a part of the capacitive element, much like in the present design. Unlike in the system presented in this work, in
[15] the input voltage, current and phase shift were measured before the coupler, however with well-characterized transfer function and power dissipated in the coupler. After measuring the reflected power and subtracting it from the total dissipated 
power, the results showed the overall reproducibility of the order of $10 \%$, which is also the higher limit of coupler losses induced by the temperature-related drift and the level of reflected power.

\section{Conclusions, comments and outlook}

This paper presents a multiplying probe for accurate power measurements in the $\mathrm{mW}-\mathrm{W}$ range on an RF-driven atmospheric pressure plasma jet, fully integrated on a miniature PCB and implemented between the power coupler and the electrodes.

The implemented crosstalk cancellation mechanism is contingent upon the elements of the electronic circuit that cause crosstalk being predictable and reproducible; in the presented design this is achieved by integrating the entire signal processing including multiplication into a miniaturized PCB. This is also the main advantage over the stateof-the-art in digital data collection and further multiplication of signals. Even though the digital data collection approach can in theory offer more accuracy, the catch is in obtaining correct signals. When using a digital sampling oscilloscope for signal collection and processing, it is unavoidable for the voltage probe not to pick up some signal related to circuit current. Even the smallest loop in the probe-tip's return path will generate an unwanted EM field related to circuit currents. Multiplying this EM field with the signal from the current sensor will result in false auto correlation results (multiplication of current with itself), and the same goes for voltage-related content picked up by the current sensor (e.g. capacitive coupling and finite common mode rejection). If the probes are separate units, which commercial probes are, then these cross-talk components will also be dependent on orientation of the probes and difficult to predict or reproduce. The magnitude of the false autocorrelation result is not necessarily proportional to the magnitude of real power nor is it constant, which means that it cannot be simply factored or zeroed out. This error manifests itself as perceived measured power proportional to voltage or current squared even if no real load is present.

Temperature compensation system is added to the design to compensate for the errors caused by the heating of the electronics during operation.

The probe features three outputs-one from the multiplier reflecting the measured power, and two showing the current and the voltage signals as measured at the electrodes, containing higher harmonics as well as the signal at $13.56 \mathrm{MHz}$.

The probe measures the input power with less than $2 \%$ deviation from the real value in the tested power range. When applied to the COST-RMJ, the main cause for deviations between subsequent measurements comes from fluctuations in the plasma.

Finally, power measurements on the COST-RMJ have been presented in the range between 160 and $320 \mathrm{~V}$ RMS, where it has been determined that there is between $0.1 \mathrm{~W}$ and $1.4 \mathrm{~W}$ dissipated in the plasma in $5.0 \mathrm{He}$ with addition of $0.5 \%$ of $\mathrm{O}_{2}$. The results agree with those previously published [15].
In conclusion, the advantage of the probe presented in this work is its higher accuracy, the implemented cross-talk cancellation which is essential for accurate power measurements and the fact that it produces an instantaneous output signal, directly representing power without a no-plasma reference measurement, including the fundamental as well as higher harmonics. This could be used for real-time power monitoring (e.g. when modulating the power), to automatically control or stabilize the power level and to examine the relative relationship of harmonics as a function of operating parameters of the COST-RMJ.

\section{Acknowledgments}

The authors would like to thank J Moerel of the Equipment \& Prototype Center at Eindhoven University of Technology for inspiring this work and Professor Nicholas St John Braithwaite of The Open University, UK and Dr Volker Schulz-von der Gathen of Ruhr Universität Bochum, Germany for stimulating discussions and for providing the COST-RMJ device. This work is a part of the COST action MP1101 Biomedical Applications of Atmospheric Pressure Plasma Technology.

\section{References}

[1] Stoffels E, Flikweert A J, Stoffels W W and Kroesen G M W 2002 Plasma Sources Sci. Technol. 11383

[2] Iza F, Kim G J, Lee S M, Lee J K, Walsh J L, Zhang Y T and Kong M G 2008 Plasma Process. Polym. 5322

[3] Kong M G, Kroesen G, Morfill G, Nosenko T, Shimizu T, van Dijk J and Zimmermann J L 2009 New J. Phys. 11115012

[4] von der Gathen V S, Buck V, Gans T, Knake N, Niemi K, Reuter S, Schaper L and Winter J 2007 Contrib. Plasma Phys. $\mathbf{4 7} 510$

[5] Niemi K, Reuter S, Schaper L, Knake N, von der Gathen V S and Gans T 2007 J. Phys.: Conf. Ser. 71012012

[6] Knake N, Reuter S, Niemi K, von der Gathen V S and Winter J 2008 J. Phys. D: Appl. Phys. 41194006

[7] von der Gathen V S, Schaper L, Knake N, Reuter S, Niemi K, Gans T and Winter J 2008 J. Phys. D: Appl. Phys. 41194004

[8] Schaper L, Reuter S, Waskoenig J, Niemi K, von der Gathen V S and Gans T 2009 J. Phys.: Conf. Ser. 162012013

[9] Reuter S, Niemi K, von der Gathen V S and Dobele H F 2009 Plasma Sources Sci. Technol. 18015006

[10] Knake N and von der Gathen V S 2010 Eur. Phys. J. D 60645

[11] Ellerweg D, Benedikt J, von Keudell A, Knake N and von der Gathen V S 2010 New J. Phys. 12013021

[12] Waskoenig J, Niemi K, Knake N, Graham L M, Reuter S, von der Gathen V S and Gans T 2010 Plasma Sources Sci. Technol. 19045018

[13] Bibinov N, Knake N, Bahre H, Awakowicz P and von der Gathen V S 2011 J. Phys. D: Appl. Phys. 44345204

[14] Schroder D, Bahre H, Knake N, Winter J, de los Arcos T and von der Gathen V S 2012 Plasma Sources Sci. Technol. 21024007

[15] Marinov D and Braithwaite N S J 2014 Plasma Sources Sci. Technol. 23062005

[16] Golda J et al 2016 J. Phys. D: Appl. Phys. 49084003

[17] Léveillé V and Coulombe S 2006 Meas. Sci. Technol. 173027 